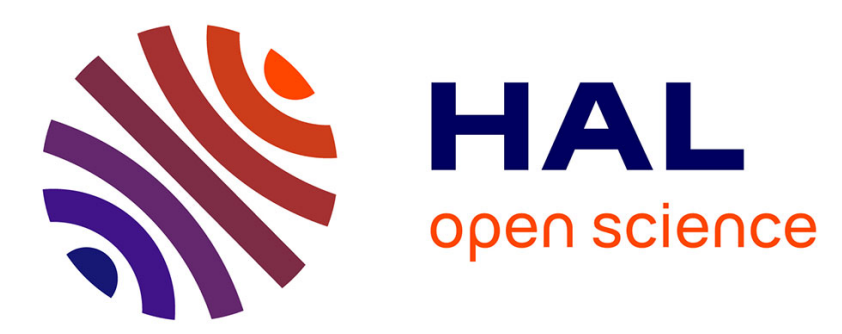

\title{
Twist Feasibility Analysis of Cable-Driven Parallel Robots
}

\author{
Saman Lessanibahri, Marc Gouttefarde, Stéphane Caro, Philippe Cardou
}

\section{To cite this version:}

Saman Lessanibahri, Marc Gouttefarde, Stéphane Caro, Philippe Cardou. Twist Feasibility Analysis of Cable-Driven Parallel Robots. 3rd International Conference on Cable-Driven Parallel Robots, Aug 2017, Québec, Canada. pp.128-139, 10.1007/978-3-319-61431-1_12 . hal-01757798

\section{HAL Id: hal-01757798 \\ https://hal.science/hal-01757798}

Submitted on 4 Apr 2018

HAL is a multi-disciplinary open access archive for the deposit and dissemination of scientific research documents, whether they are published or not. The documents may come from teaching and research institutions in France or abroad, or from public or private research centers.
L'archive ouverte pluridisciplinaire HAL, est destinée au dépôt et à la diffusion de documents scientifiques de niveau recherche, publiés ou non, émanant des établissements d'enseignement et de recherche français ou étrangers, des laboratoires publics ou privés. 


\title{
Twist Feasibility Analysis of Cable-Driven Parallel Robots
}

\author{
S. Lessanibahri ${ }^{1}$, M. Gouttefarde ${ }^{2}$, S. Caro ${ }^{3}$, and P. Cardou ${ }^{4}$
}

\begin{abstract}
Although several papers addressed the wrench capabilities of cable-driven parallel robots (CDPRs), few have tackled the dual question of their twist capabilities. In this paper, these twist capabilities are evaluated by means of the more specific concept of twist feasibility, which was defined by Gagliardini et al. in a previous work. A CDPR posture is called twist-feasible if all the twists (point-velocity and angular-velocity combinations), within a given set, can be produced at the CDPR mobile platform, within given actuator speed limits. Two problems are solved in this paper: (1) determining the set of required cable winding speeds at the CDPR winches being given a prescribed set of required mobile platform twists; and (2) determining the set of available twists at the CDPR mobile platform from the available cable winding speeds at its winches. The solutions to both problems can be used to determine the twist feasibility of $n$-degree-of-freedom (DOF) CDPRs driven by $m \geq n$ cables. An example is presented, where the twist-feasible workspace of a simple CDPR with $n=2$ DOF and driven by $m=3$ cables is computed to illustrate the proposed method.
\end{abstract}

\section{Introduction}

A cable-driven parallel robot (CDPR) consists of a base frame, a mobile platform, and a set of cables connecting in parallel the mobile platform to the base frame.

\footnotetext{
${ }^{1}$ École Centrale de Nantes, Laboratoire des Sciences du Numérique de Nantes, UMR CNRS 6004, 1, rue de la Noë, 44321 Nantes, France, e-mail: Saman.Lessanibahri@irccyn.ec-nantes.fr

2 Laboratoire d'Informatique, de Robotique et de Micro-électronique de Montpellier (LIRMM), UM - CNRS, 161 rue Ada, 34095 Montpellier Cedex 5, France, e-mail: marc.gouttefarde@lirmm.fr

${ }^{3}$ CNRS, Laboratoire des Sciences du Numérique de Nantes, UMR CNRS 6004, 1, rue de la Noë, 44321 Nantes, France, e-mail: stephane.caro@1s2n.fr

${ }^{4}$ Laboratoire de robotique, Département de génie mécanique, Université Laval, Québec, QC, Canada, e-mail: pcardou@gmc.ulaval.ca
} 
The cable lengths or tensions can be adjusted by means of winches and a number of pulleys may be used to route the cables from the winches to the mobile platform. Among other advantages, CDPRs with very large workspaces, e.g. [12, 17], heavy payloads capabilities [1], or reconfiguration capabilities, e.g. [8, 21] can be designed. Moreover, the moving parts of CDPRs being relatively light weight, fast motions of the mobile platform can be obtained, e.g. [15].

The cables of a CDPR can only pull and not push on the mobile platform and their tension shall not become larger than some maximum admissible value. Hence, for a given mobile platform pose, the determination of the feasible wrenches at the platform is a fundamental issue, which has been the subject of several previous works, e.g. $[3,13]$. A relevant issue is then to determine the set of wrench feasible poses, i.e., the so-called Wrench-Feasible Workspace (WFW) [2, 19], since the shape and size of the latter highly depends on the cable tension bounds and on the CDPR geometry [22]. Another issue which may strongly restrict the usable workspace of a CDPR or, divide it into several disjoint parts, are cable interferences. Therefore, software tools allowing the determination of the interference-free workspace and of the WFW have been proposed, e.g. [4, 18],. Besides, recently, a study on acceleration capabilities was proposed in $[5,9]$.

As noted in [7] and as well known, in addition to wrench feasibility, the design of the winches of a CDPR also requires the consideration of cable and mobile platform velocities since the selection of the winch characteristics (motors, gearboxes, and drums) has to deal with a trade-off between torque and speed. Twist feasibility is then the study of the relationship between the feasible mobile platform twists (linear and angular velocities) and the admissible cable coiling/uncoiling speeds. In the following, the cable coiling/uncoiling speeds are loosely referred to as cable velocities. The main purpose of this paper is to clarify the analysis of twist feasibility and of the related twist-feasible workspace proposed in [7]. Contrary to [7], the twist feasibility analysis proposed here is based on the usual CDPR differential kinematics where the Jacobian matrix maps the mobile platform twist into the cable velocities. This approach is most important for redundantly actuated CDPRs, whose Jacobian matrix is rectangular.

A number of concepts in this paper are known, notably from manipulability ellipsoids of serial robots, e.g. [23], and from studies on the velocity performance of parallel robots, e.g. [16]. A review of these works is however out of the scope of the present paper whose contribution boils down to a synthetic twist feasibility analysis of $n$-degrees-of-freedom (DOF) CDPRs driven by $m$ cables, with $m \geq n$. The CDPR can be fully constrained or not, and the cable mass and elasticity are neglected.

The paper is organized as follows. The usual CDPR wrench and Jacobian matrices are defined in Section 2. Section 3 presents the twist feasibility analysis, which consists in solving two problems. The first one is the determination of the set of cable velocities corresponding to a given set of required mobile platform twists (Section 3.1). The second problem is the opposite since it is defined as the calculation of the set of mobile platform twists corresponding to a given set of cable velocities (Section 3.2). The twist and cable velocity sets considered in this paper are convex 


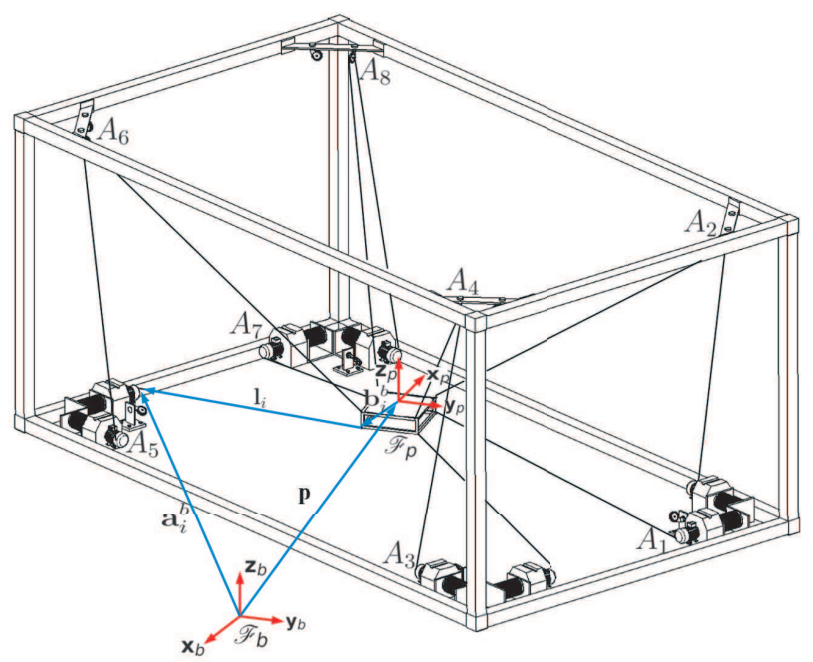

Fig. 1: Geometric description of a fully constrained CDPR

polytopes. In Section 4, a 2-DOF point-mass CDPR driven by 3 cables is considered to illustrate the twist feasibility analysis. Section 5 concludes the paper.

\section{Wrench and Jacobian Matrices}

In this section, the well-known wrench matrix and Jacobian matrix of $n$-DOF $m$ cable CDPRs are defined. The wrench matrix maps the cable tensions into the wrench applied by the cables on the CDPR mobile platform. The Jacobian matrix relates the time derivatives of the cable lengths to the twist of the mobile platform. These two matrices are essentially the same since one is minus the transpose of the other.

Some notations and definitions are first introduced. As illustrated in Fig. 1, let us consider a fixed reference frame, $\mathscr{F}_{b}$, of origin $O_{b}$ and axes $\mathbf{x}_{b}, \mathbf{y}_{b}$ and $\mathbf{z}_{b}$. The coordinate vectors ${ }^{b} \mathbf{a}_{i}, i=1, \ldots, m$ define the positions of the exit points, $A_{i}, i=1, \ldots, m$, with respect to frame $\mathscr{F}_{b} . A_{i}$ is the point where the cable exits the base frame and extends toward the mobile platform. In this paper, the exit points $A_{i}$ are assumed to be fixed, i.e., the motion of the output pulleys is neglected. A frame $\mathscr{F}_{p}$, of origin $O_{p}$ and axes $\mathbf{x}_{p}, \mathbf{y}_{p}$ and $\mathbf{z}_{p}$, is attached to the mobile platform. The vectors ${ }^{p} \mathbf{b}_{i}, i=1, \ldots, m$ are the position vectors of the points $B_{i}$ in $\mathscr{F}_{p}$. The cables are attached to the mobile platform at points $B_{i}$.

The vector ${ }^{b} \mathbf{l}_{i}$ from $B_{i}$ to $A_{i}$ is given by

$$
{ }^{b} \mathbf{l}_{i}={ }^{b} \mathbf{a}_{i}-\mathbf{p}-\mathbf{R}^{p} \mathbf{b}_{i}, i=1, \ldots, m
$$


where $\mathbf{R}$ is the rotation matrix defining the orientation of the mobile platform, i.e., the orientation of $\mathscr{F}_{p}$ in $\mathscr{F}_{b}$, and $\mathbf{p}$ is the position vector of $\mathscr{F}_{p}$ in $\mathscr{F}_{b}$. The length of the straight line segment $A_{i} B_{i}$ is $l_{i}=\left\|{ }^{b} \mathbf{l}_{i}\right\|_{2}$ where $\|\cdot\|_{2}$ is the Euclidean norm. Neglecting the cable mass, $l_{i}$ corresponds to the length of the cable segment from point $A_{i}$ to point $B_{i}$. Moreover, neglecting the cable elasticity, $l_{i}$ is the "active" length of the cable that should be unwound from the winch drum. The unit vectors along the cable segment $A_{i} B_{i}$ is given by

$$
{ }^{b} \mathbf{d}_{i}={ }^{b} \mathbf{l}_{i} / l_{i}, i=1, \ldots, m
$$

Since the cable mass is neglected in this paper, the force applied by the cable on the platform is equal to $\tau_{i}^{b} \mathbf{d}_{i}, \tau_{i}$ being the cable tension. The static equilibrium of the CDPR platform can then be written $[14,20]$

$$
\mathbf{W} \tau+\mathbf{w}_{e}=0
$$

where $\mathbf{w}_{e}$ is the external wrench acting on the platform, $\tau=\left[\tau_{1}, \ldots, \tau_{m}\right]^{\mathrm{T}}$ is the vector of cable tensions, and $\mathbf{W}$ is the wrench matrix. The latter is an $n \times m$ matrix defined as

$$
\mathbf{W}=\left[\begin{array}{cccc}
{ }^{b} \mathbf{d}_{1} & { }^{b} \mathbf{d}_{2} & \ldots & { }^{b} \mathbf{d}_{m} \\
\mathbf{R}^{p} \mathbf{b}_{1} \times{ }^{b} \mathbf{d}_{1} & \mathbf{R}^{p} \mathbf{b}_{2} \times{ }^{b} \mathbf{d}_{2} & \ldots & \mathbf{R}^{p} \mathbf{b}_{m} \times{ }^{b} \mathbf{d}_{m}
\end{array}\right]
$$

The differential kinematics of the CDPR establishes the relationship between the twist $\mathbf{t}$ of the mobile platform and the time derivatives of the cable lengths $\mathbf{i}$

$$
\mathbf{J t}=\mathbf{i}
$$

where $\mathbf{J}$ is the $m \times n$ Jacobian matrix and $\mathbf{i}=\left[\dot{l}_{1}, \ldots, \dot{l}_{m}\right]^{\mathrm{T}}$. The twist $\mathbf{t}=[\dot{\mathbf{p}}, \boldsymbol{\omega}]^{T}$ is composed of the velocity $\dot{\mathbf{p}}$ of the origin of frame $\mathscr{F}_{p}$ with respect to $\mathscr{F}_{b}$ and of the angular velocity $\boldsymbol{\omega}$ of the mobile platform with respect to $\mathscr{F}_{b}$. Moreover, the well-known kineto-statics duality leads to

$$
\mathbf{J}=-\mathbf{W}^{T}
$$

In the remainder of this paper, $\mathbf{i}$ is loosely referred to as cable velocities. The wrench and Jacobian matrices depend on the geometric parameters $\mathbf{a}_{i}$ and $\mathbf{b}_{i}$ of the CDPR and on the mobile platform pose, namely on $\mathbf{R}$ and $\mathbf{p}$.

\section{Twist Feasibility Analysis}

This section contains the contribution of the paper, namely, a twist feasibility analysis which consists in solving the following two problems.

1. For a given pose of the mobile platform of a CDPR and being given a set $[\mathbf{t}]_{r}$ of required mobile platform twists, determine the corresponding set of cable velocities $\mathbf{i}$. The set of cable velocities to be determined is called the Required Cable 
Velocity Set (RCVS) and is denoted $[\mathbf{i}]_{r}$. The set $[\mathbf{t}]_{r}$ is called the Required Twist Set (RTS).

2. For a given pose of the mobile platform of a CDPR and being given a set $[\mathbf{i}]_{a}$ of available (admissible) cable velocities, determine the corresponding set of mobile platform twists $\mathbf{t}$. The former set, $[\mathbf{i}]_{a}$, is called the Available Cable Velocity Set (ACVS) while the latter is denoted $[\mathbf{t}]_{a}$ and called the Available Twist Set (ATS).

In this paper, the discussion is limited to the cases where both the RTS $[\mathbf{t}]_{r}$ and the ACVS $[\mathrm{i}]_{a}$ are convex polytopes.

Solving the first problem provides the RCVS from which the maximum values of the cable velocities required to produce the given RTS $[\mathbf{t}]_{r}$ can be directly deduced. If the winch characteristics are to be determined, the RCVS allows to determine the required speeds of the CDPR winches. If the winch characteristics are already known, the RCVS allows to test whether or not the given RTS is feasible.

Solving the second problem provides the ATS which is the set of twists that can be produced at the mobile platform. It is thus useful either to determine the velocity capabilities of a CDPR or to check whether or not a given RTS is feasible.

Note that the feasibility of a given RTS can be tested either in the cable velocity space, by solving the first problem, or in the space of platform twists, by solving the second problem. Besides, note also that the twist feasibility analysis described above does not account for the dynamics of the CDPR.

\subsection{Problem 1: Required Cable Velocity Set (RCVS)}

The relationship between the mobile platform twist $\mathbf{t}$ and the cable velocities $\mathbf{I}$ is the differential kinematics in (5). According to this equation, the RCVS $[\mathrm{i}]_{r}$ is defined as the image of the convex polytope $[\mathbf{t}]_{r}$ under the linear map $\mathbf{J}$. Consequently, $[\mathbf{i}]_{r}$ is also a convex polytope [24].

Moreover, if $[\mathbf{t}]_{r}$ is a box, the RCVS $[\mathbf{i}]_{r}$ is a particular type of polytope called a zonotope. Such a transformation of a box into a zonotope has previously been studied in CDPR wrench feasibility analysis $[3,10,11]$. Indeed, a box of admissible cable tensions is mapped by the wrench matrix $\mathbf{W}$ into a zonotope in the space of platform wrenches. However, a difference lies in the dimensions of the matrices $\mathbf{J}$ and $\mathbf{W}$, $\mathbf{J}$ being of dimensions $m \times n$ while $\mathbf{W}$ is an $n \times m$ matrix, where $n \leq$ $m$. When $n<m$, on the one hand, $\mathbf{W}$ maps the $m$-dimensional box of admissible cable tensions into the $n$-dimensional space of platform wrenches. On the other hand, $\mathbf{J}$ maps $n$-dimensional twists into its range space which is a linear subspace of the $m$-dimensional space of cable velocities $\mathbf{i}$. Hence, when $\mathbf{J}$ is not singular, the $n$-dimensional box $[\mathbf{t}]_{r}$ is mapped into the zonotope $[\mathbf{i}]_{r}$ which lies into the $n$ dimensional range space of $\mathbf{J}$, as illustrated in Fig. 3 . When $\mathbf{J}$ is singular and has rank $r, r<n$, the $n$-dimensional box $[\mathbf{t}]_{r}$ is mapped into a zonotope of dimension $r$.

When an ACVS $[\mathbf{i}]_{a}$ is given, a pose of the mobile platform of a CDPR is twist feasible if 


$$
[\mathbf{i}]_{r} \subseteq[\mathbf{i}]_{a}
$$

Since $[\mathbf{i}]_{a}$ is a convex polytope, (7) is verified whenever all the vertices of $[\mathbf{i}]_{r}$ are included in $[\mathbf{i}]_{a}$. Moreover, it is not difficult to prove that $[\mathbf{i}]_{r}$ is the convex hull of the images under $\mathbf{J}$ of the vertices of $[\mathbf{t}]_{r}$. Hence, a simple method to verify if a CDPR pose is twist feasible consists in verifying whether the images of the vertices of $[\mathbf{t}]_{r}$ are all included into $[\mathbf{i}]_{a}$.

\subsection{Problem 2: Available Twist Set (ATS)}

The problem is to determine the ATS $[\mathbf{t}]_{a}$ corresponding to a given ACVS $[\mathbf{i}]_{a}$.

In the most general case considered in this paper, $[\mathbf{i}]_{a}$ is a convex polytope. By the Minkowski-Weyl's Theorem, a polytope can be represented as the solution set of a finite set of linear inequalities, the so-called (halfspace) $\mathrm{H}$-representation of the polytope $[6,24]$, i.e.

$$
[\mathbf{i}]_{a}=\{\mathbf{i} \mid \mathbf{C i} \leq \mathbf{d}\}
$$

where matrix $\mathbf{C}$ and vector $\mathbf{d}$ are assumed to be known.

According to (5), the ATS is defined as

$$
[\mathbf{t}]_{a}=\left\{\mathbf{t} \mid \mathbf{J t} \in[\mathbf{i}]_{a}\right\}
$$

which, using (8), implies that

$$
[\mathbf{t}]_{a}=\{\mathbf{t} \mid \mathbf{C J} \mathbf{t} \leq \mathbf{d}\}
$$

The latter equation provides an H-representation of the ATS $[\mathbf{t}]_{a}$.

In practice, when the characteristics of the winches of a CDPR are known, the motor maximum speeds limit the set of possible cable velocities as follows

$$
\dot{l}_{i, \min } \leq \dot{l}_{i} \leq \dot{i}_{i, \max }
$$

where $\dot{l}_{i, \min }$ and $\dot{l}_{i, \max }$ are the minimum and maximum cable velocities. Note that, usually, $\dot{l}_{i, \min }=-\dot{l}_{i, \max }, \dot{l}_{1, \min }=\dot{l}_{2, \min }=\ldots=\dot{l}_{m, \min }$, and $\dot{l}_{1, \max }=\dot{l}_{2, \max }=\ldots=\dot{l}_{m, \max }$. In other words, $\mathbf{C}$ and $\mathbf{d}$ in (8) are defined as

$$
\mathbf{C}=\left[\begin{array}{r}
\mathbf{1} \\
-\mathbf{1}
\end{array}\right] \quad \text { and } \quad \mathbf{d}=\left[i_{1, \max }, \ldots, i_{m, \max },-\dot{l}_{1, \min }, \ldots,-\dot{l}_{m, \min }\right]^{T}
$$

where $\mathbf{1}$ is the $m \times m$ identity matrix. Eq. (10) can then be written as follows

$$
[\mathbf{t}]_{a}=\left\{\mathbf{t} \mid \mathbf{i}_{\min } \leq \mathbf{J t} \leq \mathbf{i}_{\max }\right\}
$$

where $\mathbf{i}_{\text {min }}=\left[i_{1, \min }, \ldots, i_{m, \min }\right]^{T}$ and $\dot{\mathbf{i}}_{\max }=\left[i_{1, \max }, \ldots, i_{m, \max }\right]^{T}$. 
When a RTS $[\mathbf{t}]_{r}$ is given, a pose of the mobile platform of a CDPR is twist feasible if

$$
[\mathbf{t}]_{r} \subseteq[\mathbf{t}]_{a}
$$

In this paper, $[\mathbf{t}]_{r}$ is assumed to be a convex polytope. Hence, (14) is verified whenever all the vertices of $[\mathbf{t}]_{r}$ are included in $[\mathbf{t}]_{a}$. With the H-representation of $[\mathbf{t}]_{a}$ in (10) (or in (13)), testing if a pose is twist feasible amounts to verifying if all the vertices of $[\mathbf{t}]_{r}$ satisfy the inequality system in (10) (or in (13)). Testing twist feasibility thereby becomes a simple task as soon as the vertices of $[\mathbf{t}]_{r}$ are known.

Finally, let the twist feasible workspace (TFW) of a CDPR be the set of twist feasible poses of its mobile platform. It is worth noting that the boundaries of the TFW are directly available in closed form from (10) or (13). If the vertices of the (convex) RTS are denoted $\mathbf{t}_{j}, j=1, \ldots, k$, and the rows of the Jacobian matrix are $-\mathbf{w}_{i}^{T}$, according to (13), the TFW is defined by $\dot{l}_{i, \min } \leq-\mathbf{w}_{i}^{T} \mathbf{t}_{j}$ and $-\mathbf{w}_{i}^{T} \mathbf{t}_{j} \leq \dot{l}_{i, \max }$, for all possible combinations of $i$ and $j$. Since $\mathbf{w}_{i}$ contains the only variables in these inequalities that depend on the mobile platform pose, and because the closedform expression of $\mathbf{w}_{i}$ as a function of the pose is known, the expressions of the boundaries of the TFW are directly obtained.

\section{Case Study}

This section deals with the twist feasibility analysis of the two-DOF point-mass planar CDPR driven by three cables shown in Fig.2. The robot is $3.5 \mathrm{~m}$ long and $2.5 \mathrm{~m}$ high. The three exit points of the robot are named $A_{1}, A_{2}$ are $A_{3}$, respectively. The point-mass is denoted $P .{ }^{b} \mathbf{d}_{1},{ }^{b} \mathbf{d}_{2}$ and ${ }^{b} \mathbf{d}_{3}$ are the unit vectors, expressed in frame $\mathscr{F}_{b}$, of the vectors pointing from point-mass $P$ to cable exit points $A_{1}, A_{2}$ are $A_{3}$, respectively. The $3 \times 2$ Jacobian matrix $\mathbf{J}$ of this planar CDPR takes the form:

$$
\mathbf{J}=-\left[\begin{array}{l}
{ }^{b} \mathbf{d}_{1}^{T} \\
{ }^{b} \mathbf{d}_{2}^{T} \\
{ }^{b} \mathbf{d}_{3}^{T}
\end{array}\right]
$$

Figure 3 is obtained by solving the Problem 1 formulated in Sec. 3. For the robot configuration depicted in Fig. $3 \mathrm{a}$ and the given RTS of the point-mass $P$ represented in Fig. 3b, the RCVS for the three cables of the planar CDPR are illustrated in Figs. $3 \mathrm{c}$ to $3 \mathrm{f}$. Note that the RTS is defined as:

$$
\begin{aligned}
& -1 \mathrm{~m} . \mathrm{s}^{-1} \leq \dot{x}_{P} \leq 1 \mathrm{~m} . \mathrm{s}^{-1} \\
& -1 \mathrm{~m} . \mathrm{s}^{-1} \leq \dot{y}_{P} \leq 1 \mathrm{~m} . \mathrm{s}^{-1}
\end{aligned}
$$

where $\left[\dot{x}_{P}, \dot{y}_{P}\right]^{T}$ is the velocity of $P$ in the fixed reference frame $\mathscr{F}_{b}$.

Figure 4 depicts the isocontours of the Maximum Required Cable Velocity (MRCV) for each cable through the Cartesian space and for the RTS shown in Fig. 3b. Those results are obtained by solving Problem 1 for all positions of point $P$. It is apparent 


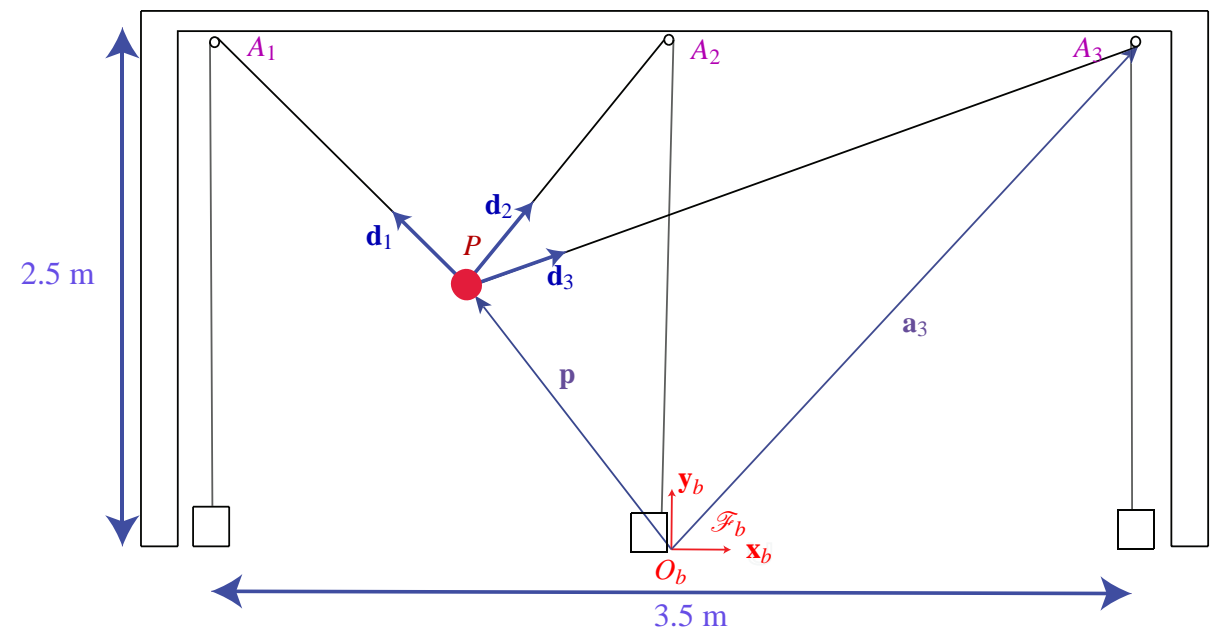

Fig. 2: A two-DOF point-mass planar cable-driven parallel robot driven by three cables

that $P$ RTS is satisfied through the Cartesian space as long as the maximum velocity of each cable is higher than $\sqrt{2} \mathrm{~m} \cdot \mathrm{s}^{-1}$, namely, $\dot{l}_{1, \max }=\dot{l}_{2, \max }=\dot{l}_{3, \max }=\sqrt{2} \mathrm{~m} \cdot \mathrm{s}^{-1}$ with $\dot{l}_{i, \min }=-\dot{l}_{i, \max }, i=1,2,3$.

For the Available Cable Velocity Set (ACVS) defined by inequalities (11) with

$$
i_{i, \max }=1.3 \mathrm{~m} \cdot \mathrm{s}^{-1}, i=1,2,3
$$

Fig. 5 is obtained by solving the Problem 2 formulated in Sec. 3 .

For the two robot configurations illustrated in Fig. $5 \mathrm{a}$ and $5 \mathrm{c}$, the Available Twist Set (ATS) associated to the foregoing ACVS is determined from Eq. (13). It is noteworthy that the ATS in each configuation in delimited by three pairs of lines normal to three cables, respectively. It turns out that the first robot configuration is twist feasible for the RTS defined by Eqs. (16) and (17) because the latter is included into the ATS as shown Fig. 5b. Conversely, the second robot configuration is not twist feasible as the RTS is partially outside the ATS as shown Fig. 5d.

Finally, Fig. 6 shows the TFW of the planar CDPR for four maximum cable velocity limits and for the RTS shown in Fig. 3b. It is apparent the all robot poses are twist feasible as soon as the cable velocity limits of the three cables are higher than $\sqrt{2} \mathrm{~m} \cdot \mathrm{s}^{-1}$. 


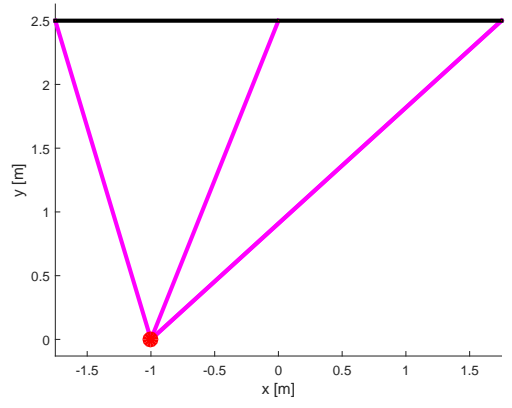

(a) Robot configuration

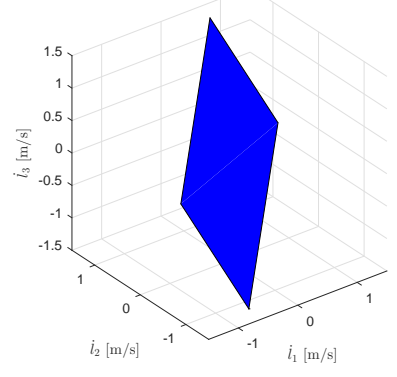

(c) Image of the RTS into the three-dimensional cable velocity space

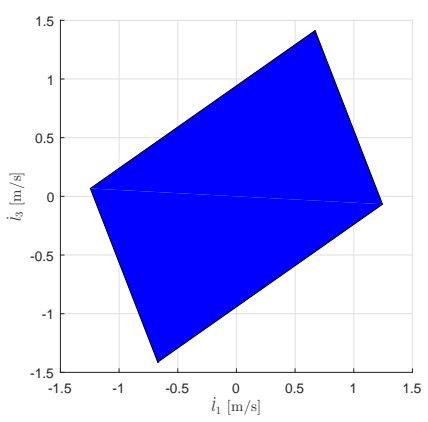

(e) Image of the RTS into the $\left(\dot{l}_{1}, \dot{l}_{3}\right)$-space

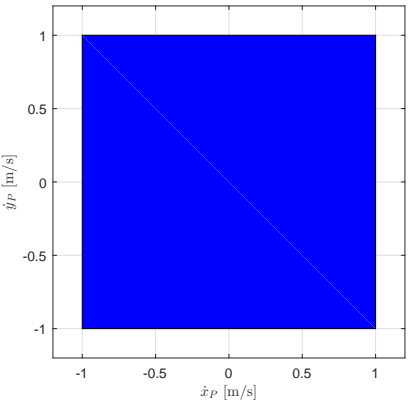

(b) RTS

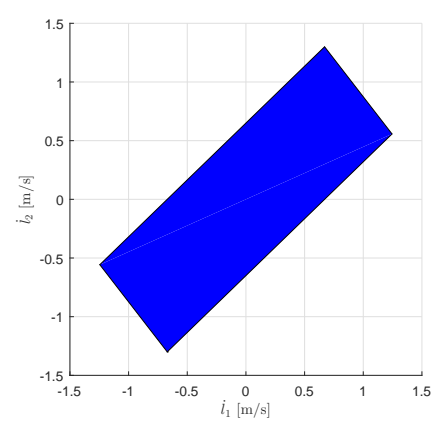

(d) Image of the RTS into the $\left(\dot{l}_{1}, \dot{i}_{2}\right)$-space

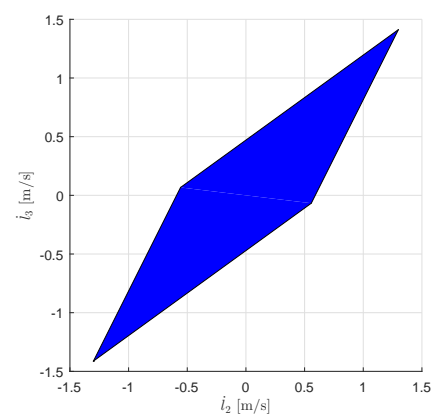

(f) Image of the RTS into the $\left(\dot{l}_{2}, \dot{l}_{3}\right)$-space

Fig. 3: Required Twist Set (RTS) of the point-mass $P$ and corresponding Required Cable Velocity Sets for the three cables of the CDPR in a given robot configuration 


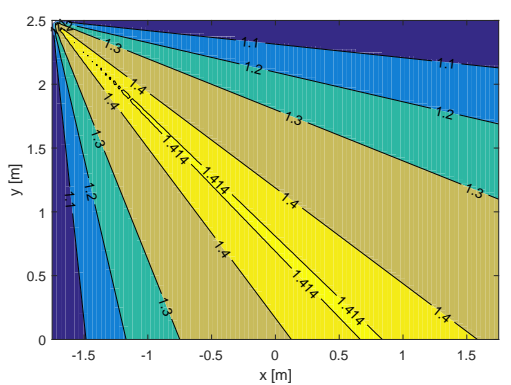

(a) Cable $1 \mathrm{MRCV}$

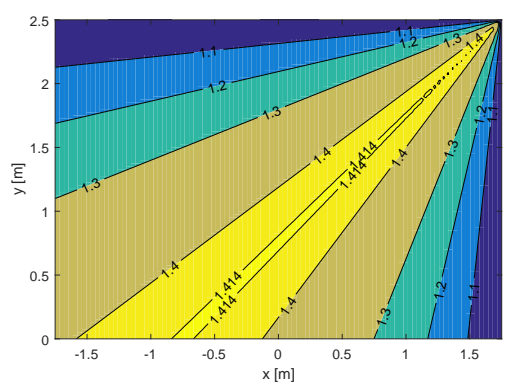

(c) Cable $3 \mathrm{MRCV}$

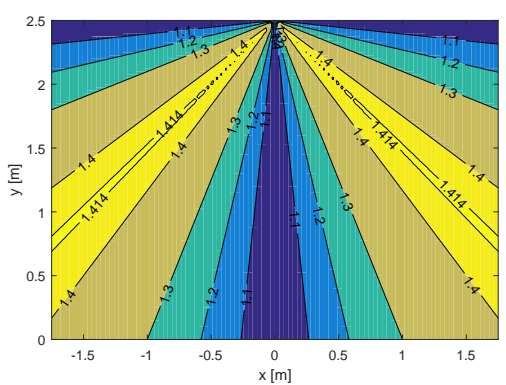

(b) Cable 2 MRCV

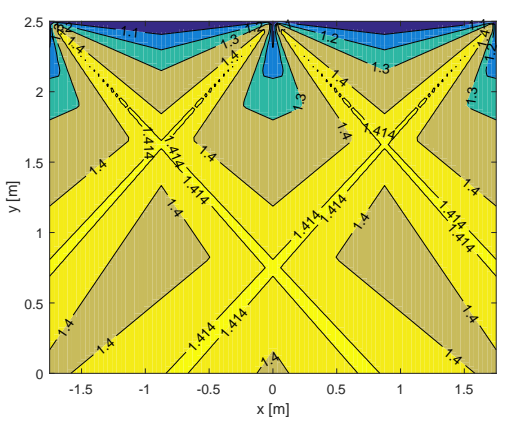

(d) Cables 1, 2 and $3 \mathrm{MRCV}$

Fig. 4: Maximum Required Cable Velocity (MRCV) of each cable through the Cartesian space for the RTS shown in Fig. 3b

\section{Conclusion}

In summary, this paper presents two methods of determining the twist-feasibility of a CDPR. The first method uses a set of required mobile platform twists to compute the corresponding required cable velocities, the latter corresponding to cable winding speeds at the winches. The second method takes the opposite route, i.e., it uses the available cable velocities to compute the corresponding set of available mobile platform twists. The second method can be applied to compute the twist-feasible workspace, i.e., to determine the set of mobile platform poses where a prescribed polyhedral required twist set is contained within the available twist set. This method can thus be used to analyze the CDPR speed capabilities over its workspace, which should prove useful in high-speed CDPR applications.

The proposed method can be seen as a dual to the one used to compute the wrench-feasible workspace of a CDPR, just as the velocity equations may be seen 


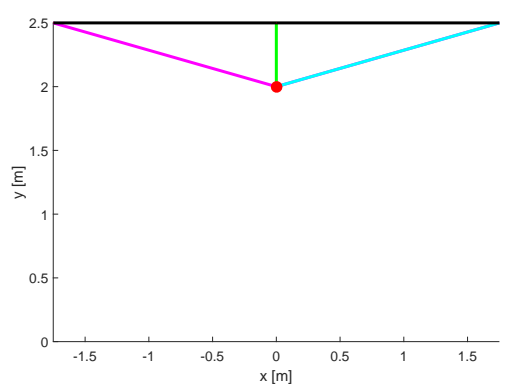

(a) A feasible twist pose

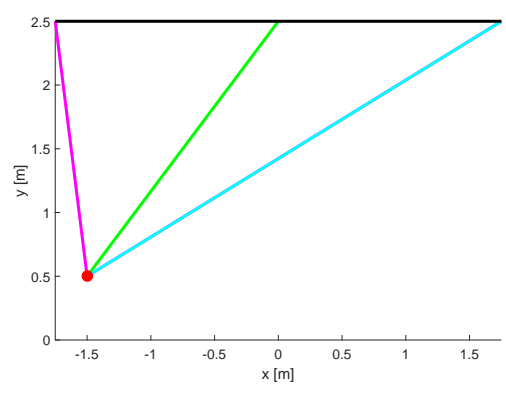

(c) An infeasible twist pose

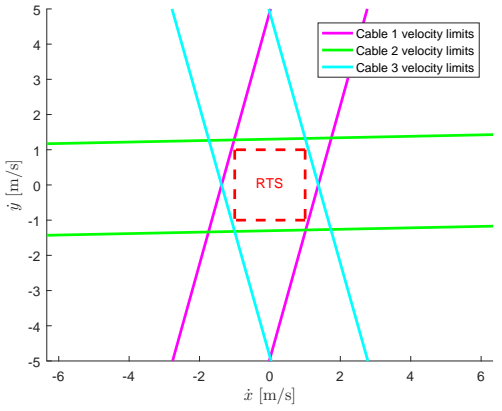

(b) The RTS is included into the ATS

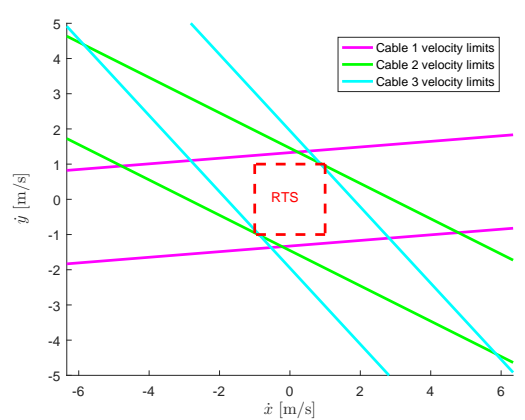

(d) The RTS is partially outside the ATS

Fig. 5: A feasible twist pose and an infeasible twist pose of the CDPR

as dual to static equations. From a mathematical standpoint, however, the problem is much simpler in the case of the twist-feasible workspace, as the feasibility conditions can be obtained explicitly. Nevertheless, the authors believe that the present paper complements nicely the previous works on wrench feasibility.

Finally, we should point out that the proposed method does not deal with the issue of guaranteeing the magnitudes of the mobile platform point-velocity or angular velocity. In such a case, the required twist set becomes a ball or an ellipsoid, and thus is no longer polyhedral. This ellipsoid could be approximated by a polytope in order to apply the method proposed in this paper. However, since the accuracy of the approximation would come at the expense of the number of conditions to be numerically verified, part of our future work will be dedicated to the problem of determining the twist-feasibility of CDPRs for ellipsoidal required twist sets.

Acknowledgements The financial support of the ANR under grant ANR-15-CE10-0006-01 (DexterWide project) is greatly acknowledged. This research work was also part of the CAROCA project managed by IRT Jules Verne (French Institute in Research and Technology in Advanced 


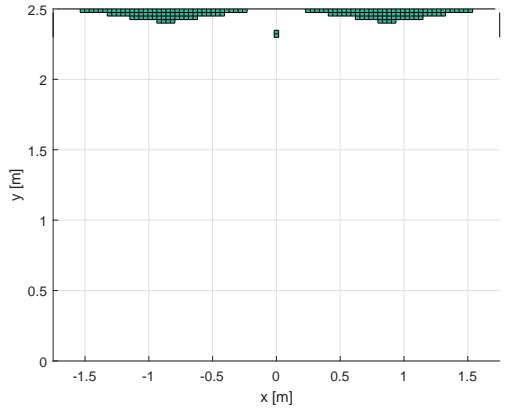

(a) $\dot{i}_{i, \max }=1.12 \mathrm{~m} \cdot \mathrm{s}^{-1}$

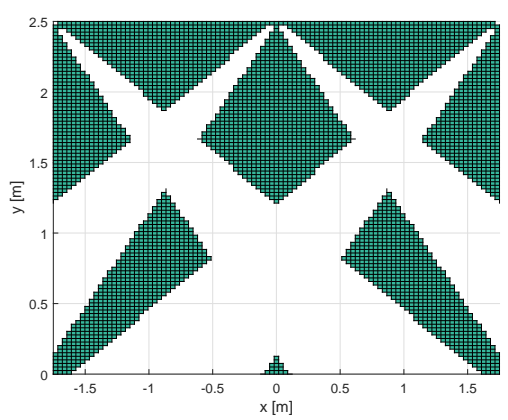

(c) $i_{i, \max }=1.4 \mathrm{~m} \cdot \mathrm{s}^{-1}$

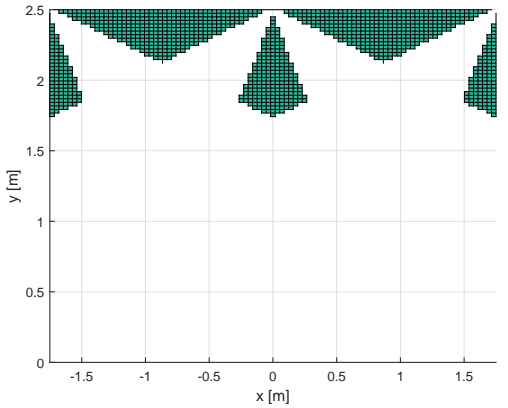

(b) $i_{i, \max }=1.32 \mathrm{~m} . \mathrm{s}^{-1}$

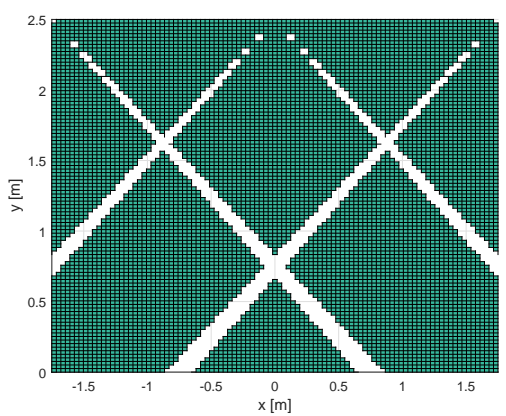

(d) $\dot{l}_{i, \max }=1.414 \mathrm{~m} \cdot \mathrm{s}^{-1}$

Fig. 6: TFW of the planar CDPR for four maximum cable velocity limits and for the RTS shown in Fig. 3b

Manufacturing Technologies for Composite, Metallic and Hybrid Structures) and of the RFI ATLANSTIC 2020 CREATOR project.

\section{References}

1. J. Albus, R. Bostelman, and N. Dagalakis. The NIST Robocrane. Journal of Robotic Systems, 10(2):709-724, 1993.

2. P. Bosscher, A. T. Riechel, and I. Ebert-Uphoff. Wrench-feasible workspace generation for cable-driven robots. IEEE Trans. on Robotics, 22(5):890-902, October 2006.

3. S. Bouchard, C. M. Gosselin, and B. Moore. On the ability of a cable-driven robot to generate a prescribed set of wrenches. ASME Journal of Mechanisms and Robotics, 2(1):1-10, 2010.

4. A. Cruz Ruiz, S. Caro, P. Cardou, and F. Guay. Arachnis : Analysis of robots actuated by cables with handy and neat interface software. In Mechanisms and Machine Science Volume, volume 32, pages 293-305. 2015. 
5. J. Eden, D. Lau, Y. Tan, and D. Oetomo. Available acceleration set for the study of motion capabilities for cable-driven robots. Mechanism and Machine Theory, 105:320-336, 2016.

6. K. Fukuda. Frequently asked questions in polyhedral computation. Technical report, http://www.ifor.math.ethz.ch/ fukuda/polyfaq/polyfaq.html.

7. L. Gagliardini, S. Caro, M. Gouttefarde, and A. Girin. Dimensioning of cable-driven parallel robot actuators, gearboxes and winches according to the twist feasible workspace. In Proc. IEEE Int. Conf. on Automation Science and Engineering, Gothenburg, Sweden, 2015.

8. L. Gagliardini, S. Caro, M. Gouttefarde, and A. Girin. Discrete reconfiguration planning for cable-driven parallel robots. Mechanism and Machine Theory, 100:313-337, 2016.

9. L. Gagliardini, M. Gouttefarde, and S. Caro. Determination of a dynamic feasible workspace for cable-driven parallel robots. In The 15th International Symposium on Advances in Robot Kinematics, Grasse, France, 2016.

10. P. Gallina, G. Rosati, and A. Rossi. 3-dof wire driven planar haptic interface. Journal of Intelligent and Robotic Systems, 32(1):23-36, 2001.

11. M. Gouttefarde and S. Krut. Characterization of Parallel Manipulator Available Wrench Set Facets. In Advanced in Robot Kinematics, Portoroz, Slovenia, 2010.

12. M. Gouttefarde, J. Lamaury, C. Reichert, and T. Bruckmann. A versatile tension distribution algorithm for $n$-DOF parallel robots driven by $n+2$ cables. IEEE Trans. on Robotics, 31(6):1444-1457, 2015.

13. M. Hassan and A. Khajepour. Analysis of Bounded Cable Tensions in Cable-Actuated Parallel Manipulators. IEEE Trans. on Robotics, 27(5):891-900, 2011.

14. M. Hiller, S. Fang, S. Mielczarek, R. Verhoeven, and D. Franitza. Design, analysis and realization of tendon-based parallel manipulators. Mech. and Mach. Theory, 40(4):429-445, April 2005.

15. S. Kawamura, H. Kino, and C. Won. High-speed manipulation by using parallel wire-driven robots. Robotica, 18:13-21, 2000.

16. S. Krut, O. Company, and F. Pierrot. Velocity performance indices for parallel mechanisms with actuation redundancy. Robotica, 22:129-139, 2004.

17. C. Lambert, M. Nahon, and D. Chalmers. Implementation of an Aerostat Positioning System With Cable Control. IEEE/ASME Transactions on Mechatronics, 12(1):32-40, 2007.

18. S. Perreault, P. Cardou, C. Gosselin, and M. Otis. Geometric determination of the interferencefree constant-orientation workspace of parallel cable-driven mechanisms. ASME Journal of Mechanisms and Robotics, 2(3), 2010.

19. A. T. Riechel and I. Ebert-Uphoff. Force-feasible workspace analysis for underconstrained point-mass cable robots. In Proc. IEEE Int. Conf. Robotics and Automation (ICRA), pages 4956-4962, New Orleans, LA, 2004.

20. R. G. Roberts and T. Graham, T.and Lippitt. On the Inverse Kinematics, Statics, and Fault Tolerance of Cable-Suspended Robots. Journal of Robotic Systems, 15(10):581-597, October 1998.

21. G. Rosati, D. Zanotto, and S. K. Agrawal. On the design of adaptive cable-driven systems. Journal of Mechanisms and Robotics, 3, 2011.

22. R. Verhoeven. Analysis of the Workspace of Tendon-Based Stewart-Platforms. PhD thesis, Univ. Duisburg-Essen, Germany, 2004.

23. T. Yoshikawa. Foundations of Robotics. MIT Press, Boston, MA, 2nd ed. edition, 1990.

24. G. M. Ziegler. Lectures on Polytopes. Springer-Verlag, Graduate Texts in Mathematics 152, 1994. 